Molecules 2008, 13, 220-229

\title{
molecules
}

ISSN 1420-3049

(C) 2008 by MDPI

www.mdpi.org/molecules

Full Paper

\section{Isolation and Identification of an Anti-tumor Component from Leaves of Impatiens balsamina}

\author{
Zhi-Shan Ding ${ }^{1}$, Fu-Sheng Jiang ${ }^{1}$, Ni-Pi Chen ${ }^{1}$, Gui-Yuan Lv ${ }^{1}$ and Cheng-Gang Zhu ${ }^{2, *}$ \\ ${ }^{1}$ Faculty of Life Science, Zhejiang Chinese Medical University, Hangzhou, 310053, P. R. China; \\ E-mails: zjtcmdzs@163.com (Z.-S. Ding); jfs1020@163.com (F.-S. Jiang), nebe323@gmail.com (N.-P. \\ Chen), lv.gy@263.net (G.-Y. Lv) \\ ${ }^{2}$ College of Life Science, Zhejiang University, Hangzhou 310029, P. R. China
}

* Author to whom correspondence should be addressed; E-mail: cgzhu@zju.edu.cn; Fax: (+86)-571-8820 6615; Tel:(+86)-571-8820 6615

Resubmitted version Received: 21 December 2007; in revised form: 18 January 2008 / Accepted: 28 January 2008 / Published: 31 January 2008

\begin{abstract}
We have previously shown that ethanol or chloroform extracts of the leaves of Impatiens balsamina (LIB) have anti-tumor activity against the human hepatocellular carcinoma cell line HepG2. The ethanol extracts were separated into five fractions according to polarity. An MTT assay indicated that two of the fractions had anti-tumor activity and that the petroleum ether fraction (PEF) was the most active. But the available quantities of both the PEF and chloroform fractions (CHF) were limited, precluding further study. The chloroform extract (CHE) shared almost all the same spots with the PEF and $\mathrm{CHF}$ and was plentiful enough to carry out further separations. Thus, the CHE was further separated into six sub-fractions (CHE1 6) by column chromatography. A MTT assay showed that only the CHE2 fraction had a strong tumor inhibition ratio $\left(\mathrm{IC}_{50}=6.47 \pm 0.05\right.$ $\mathrm{mg} / \mathrm{L}$ ), which was superior to that of curcumin $\left(\mathrm{IC}_{50}=13.95 \pm 0.11 \mathrm{mg} / \mathrm{L}\right)$. However, TLC revealed that CHE2 was not pure and still contained two more components. After further separation and purification, followed by TLC and MTT assay confirmation, the final active component was isolated and identified as 2-methoxy-1,4-naphthoquinone by m.p., UV, MS and ${ }^{13} \mathrm{C}$ - and ${ }^{1} \mathrm{H}-\mathrm{NMR}$ data. This is the first report demonstrating that 2-methoxy-1,4-naphthoquinone has intensive in vitro anti-tumor activity against HepG2 cells.
\end{abstract}


Keywords: Impatiens balsamina, anti-tumor, MTT, TLC, 2-methoxy-1,4-naphthoquinone

\section{Introduction}

The entirety of Impatiens balsamina plants (balsam, LIB) have been widely used in Traditional Chinese Medicine to treat rheumatism, isthmus and crural aches, fractures, superficial infections, fingernail inflammation, etc. [1]. Modern chemical and pharmacological studies have identified flavonol and naphthoquinone derivatives, some of which have strong antimicrobial [2], anti-anaphylaxis [3, 4], anti-inflammatory [5] as well as itch alleviating and anti-dermatitis activities, as the main chemical components of this plant [6]. Moreover, in some areas of China, people ingest this plant as a vegetable or anti-cancer herb, although there are currently no reports confirming the efficacy of this practice. A pilot study demonstrated that LIB has in vitro anti-tumor activity against the HepG2 human hepatocellular carcinoma cell line. We now report the isolation of the active fractions using a MTT assay-guided fractionation and identified the positive spots using TLC. The in vitro anti-tumor activity of the active fractions was also assessed. Finally, we purified and identified the active component of the fraction displaying the highest activity.

\section{Results and Discussion}

TLC analyses were performed using silica gel G plates. The results presented in Figure 1 show that the PEF, CHF and CHE share some of the same spots contained within the black rectangle (Figure 1-b), especially the dark and bright spots (see arrows in Figure 1-a). On the other hand, the ethyl acetate (EAF) and $n$-butanol (BUF) fractions as well as the EAF, BUF and water (WAF) ones also share some common spots, as marked by the blue and yellow rectangles, respectively. In addition, there were few common spots between those shared by the PEF, CHF and CHE groups and those shared by the EAF, BUF, and WAF fractions. These results were confirmed using several different solvent eluent systems (data not shown).

MTT assays were performed following the protocol described in the Experimental section. The results indicated that the PEF, CHF and CHE fractions all had some in vitro anti-tumor activity against HepG2 cells, with the PEF fraction possessing the strongest activity, having a cell inhibition ratio of approximately $80 \%$ at a concentration of $50 \mathrm{mg} / \mathrm{L}$, but there were too many common spots among the three of them to determine which spot active was the source of the observed activity. In contrast, the EAF, BUF and WAF fractions did not show any proliferation inhibition activity towards the HepG2 cells (data not shown). Consequently, only the PEF, CHF and CHE fractions were selected for further study. 
Figure 1. TLC display of the different fractions of LIB.
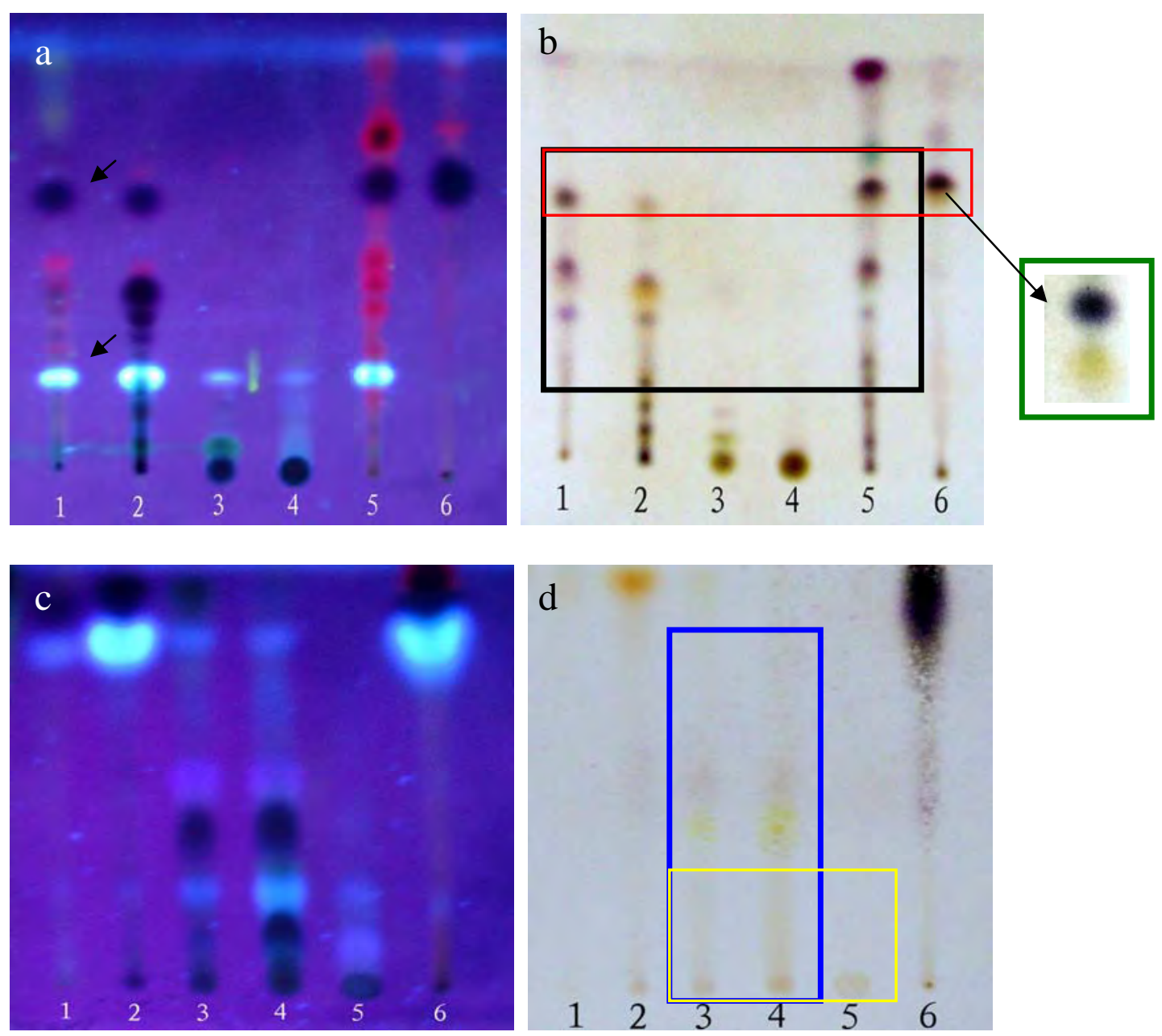

Plates $(a, b)$ were developed with chloroform-petroleum ether-methanol- formic acid = 3:4:0.5:0.1 (v/v); plates $(\mathrm{c}, \mathrm{d})$ were developed with ethyl acetate-methanol-formic acid $=6: 2: 0.1(\mathrm{v} / \mathrm{v})$. Plates $(\mathrm{a}, \mathrm{c})$ were photographed under a UV lamp (310 nm); Plates (b,d) were stained with 5\% vanillin-concentrated sulfuric acid reagents and heated at $105^{\circ} \mathrm{C}$ for $5 \mathrm{~min}$. Lanes 1-6 in a and b correspond to the PEF, CHF, EAF, BUF, CHE and CHE2 fractions, respectively. Lanes 1-6 in (c) and (d) correspond to the PEF, CHF, EAF, BUF, WAF and CHE fractions, respectively.

The dark and bright spots were the most likely candidates, as the red spots are usually caused by chlorophyll, but this still needed to be confirmed. Unfortunately, the available quantities of both PEF and CHF fractions were limited. The CHE fraction shared almost all the same spots with PEF and CHF and was plentiful enough to perform further separations. Thus, we used silica gel column chromatography and elution with a chloroform-methanol (9.5:0.5) system to further separate the CHE fraction into six sub-fractions: CHE1 CHE6 (see details in Figure 2-a). 
Figure 2. TLC analysis of CHE sub-fractions.
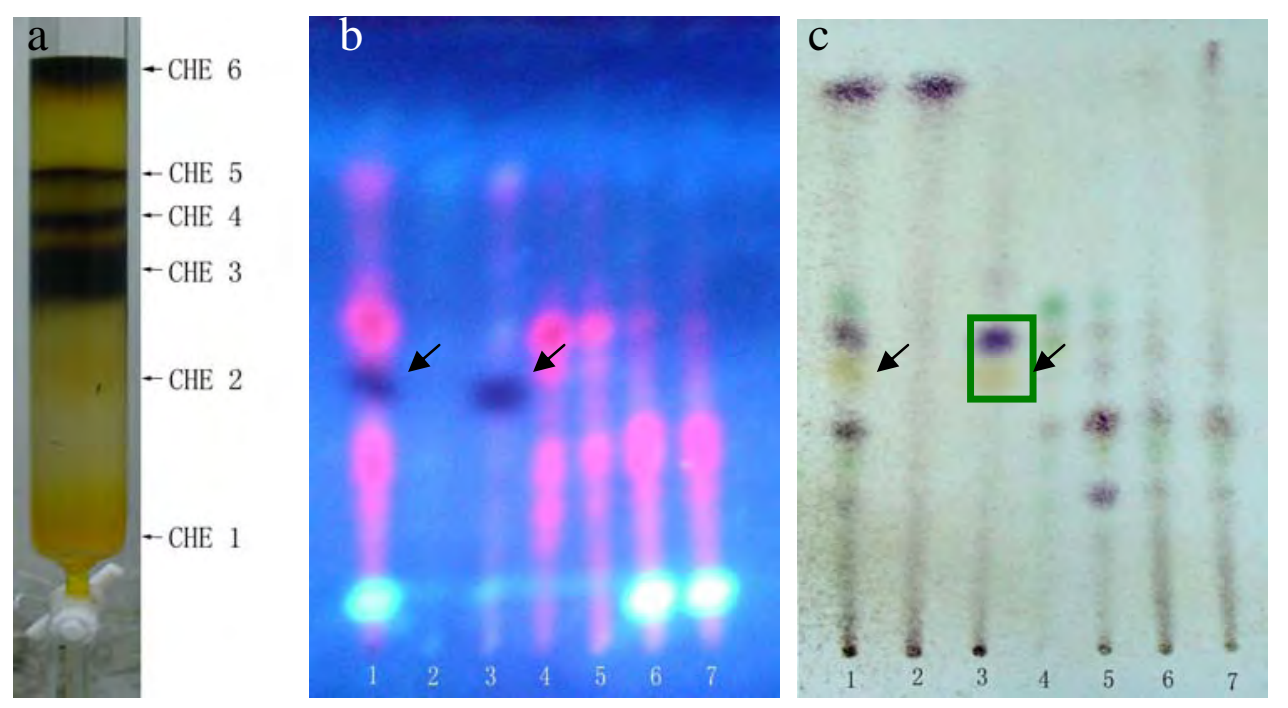

(a) Six sub-fractions of the CHE fraction separated by silica gel column chromatography. Plates (b, c) were developed with chloroform-petroleum ether-methanol-formic acid = 2:6:0.5:0.1 (v/v), but (b) was photographed under a UV lamp, while (c) was stained with $5 \%$ vanillin-concentrated sulfuric acid reagents and heated at $105^{\circ} \mathrm{C}$ for $5 \mathrm{~min}$. Lane 1 represents CHE, lanes 2-7 represented fractions CHE1 CHE6.

MTT results revealed that only the CHE2 sub-fraction exhibited inhibitory activity. Moreover, its activity was even stronger than that of the PEF fraction (Figure 3). Comparing Figure 2-b,c with Figure 1-a, b, we can easily determine that the dark spot in the red rectangle (see Figure 1-b) is the positive spot. Additionally, we confirmed that these dark spots contained two substances (black and yellow spots, as seen in the green rectangle) using two more different developer systems.

Figure 3. MTT results for the CHE sub-fractions.

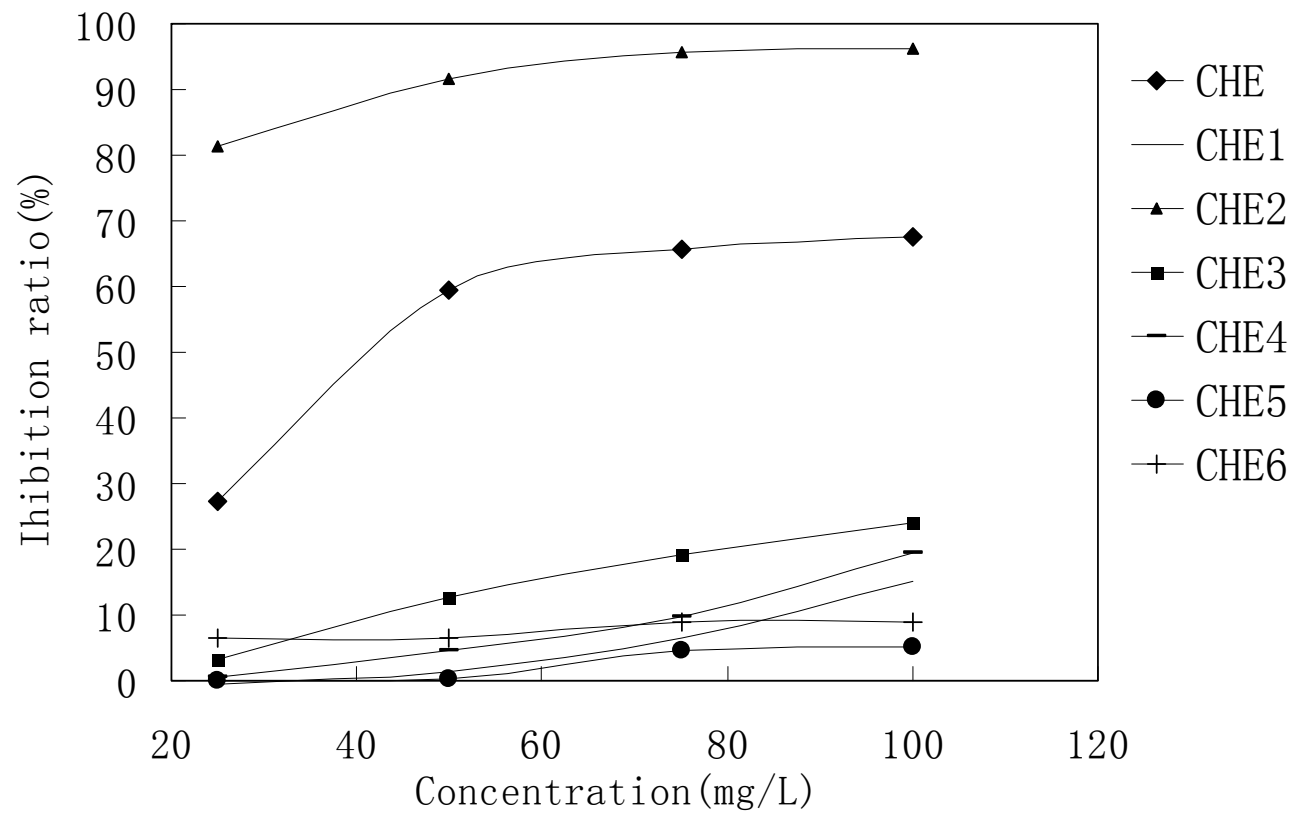


Cells in 96-wells ( $2 \times 10^{4}$ cells/well) were treated with different concentrations of CHE2 for $24 \mathrm{~h}$, and curcumin was used as a positive control. The results indicated that CHE2 possessed a strong cell inhibition effect, with an $\mathrm{IC}_{50}=6.47 \pm 0.05 \mathrm{mg} / \mathrm{L}$, as compared to curcumin $\left(\mathrm{IC}_{50}=13.95 \pm 0.11\right.$ $\mathrm{mg} / \mathrm{L}$ ). As shown in Figure 4, the inhibition effect was dose dependent.

Figure 4. Cell inhibition effect of CHE2 compared with that of curcumin.

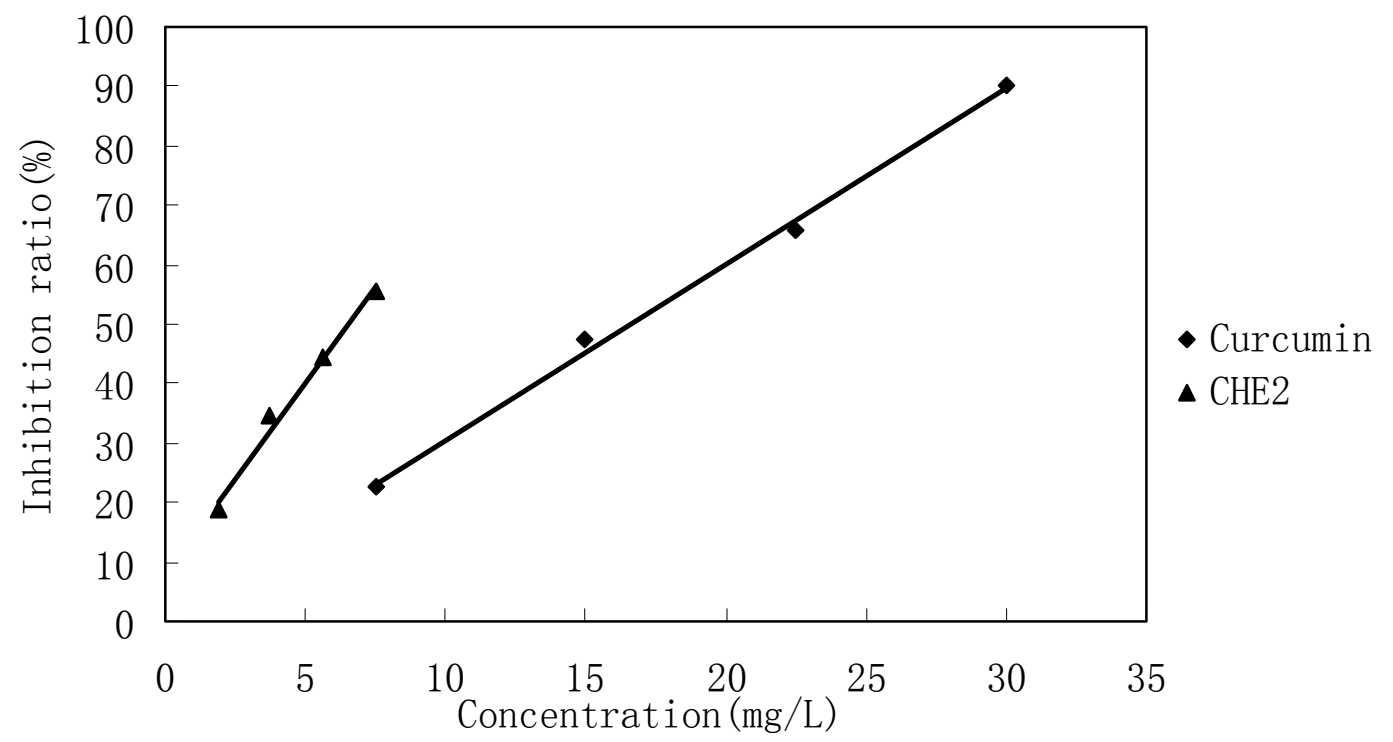

Rechromatography of fraction CHE-2 and recrystallization yielded an active single component. TLC confirmed that it corresponded to the dark spot in Figure 2-b, as marked by a black arrow. This compound was then identified as 2-methoxy-1,4-naphthoquinone (MNQ) by m.p., UV, MS and ${ }^{13} \mathrm{C}$ and ${ }^{1} \mathrm{H}$-NMR spectra. To reconfirm the anti-tumor activity of the final compound, we compared its HepG2 inhibition ratio with that of the CHE and CHE-2 fractions and curcumin. The MTT results in Table 1 show that MNQ possessed the highest activity with an $\mathrm{IC}_{50}=6.08 \pm 0.08 \mathrm{mg} / \mathrm{L}$, and that there was no significant difference with the activity of CHE2. We also determined that MNQ corresponded to $97.2 \%$ of the total CHE2 fraction,in agreement with the HPLC results (data not shown). These results concluded that MNQ is the main active component of CHE2 and with intensive anti-tumor activity against HepG2 cells in vitro.

Table 1. $\mathrm{IC}_{50}$ of the components against HepG2 cells in vitro.

\begin{tabular}{|c|c|}
\hline Components & IC $_{\mathbf{5 0}}$ (mg/L) \\
\hline CHE & $41.5 \pm 0.15$ \\
CHE2 & $6.51 \pm 0.10$ \\
MNQ & $6.08 \pm 0.08$ \\
Curcumin & $14.18 \pm 0.07$ \\
\hline
\end{tabular}

Because only the PBF and CHF fractions also contain this dark spot (Figure 1), only these fractions possess HepG2 inhibition activity. Several studies have revealed that 2-methoxy-1,4-naphthoquinone 
possesses notable antimicrobial activity. Little et al. [9] first isolated this compound from Impatiens balsamina L. and found that it had anti-fungal activity as well as low phytotoxicity. Kang [10] and Yang [2] reported that both gram-positive and gram-negative bacteria strains were sensitive to 2-methoxy-1,4-naphthoquinone. Furthermore, Yang [2] found that it was more effective than amphotericin B on seven human pathogenic fungal strains, some of which are resistant to amphotericin $\mathrm{B}$ and fluconazole. These findings validated the plant Impatiens balsamina L. as an antimicrobial herb.

Liu et al. [11] reviewed naphthoquinones and their artificial derivatives on anti-tumor activity and showed that many correlated compounds had promising future applications. Our in vitro research indicated for the first time that the 2-methoxy-1,4-naphthoquinone compound had intensive inhibition activity in HepG2 cells, with an $\mathrm{IC}_{50}$ of cell inhibition effect of $6.08 \pm 0.08 \mathrm{mg} / \mathrm{L}$. Our work implies that the Chinese herb Impatiens balsamina does indeed have some anti-tumor bioactivities. This finding will greatly benefit the clinical use of Impatiens balsamina in Chinese medicine and suggests that 2-methoxy-1, 4-naphthoquinone could be a potent anti-tumor drug candidate.

\section{Experimental}

\section{General}

Melting points were measured with a WRS-1 Digital Melting Point Apparatus [Shanghai Physical Optical Instrument Factory (SPOIF), P.R. China]. For the UV spectra 2-methoxy-1,4-naphthoquinone (1.0 mg) was dissolved in methanol and diluted to a volume of $10 \mathrm{~mL}$ with methanol, then $100 \mu \mathrm{L}$ of this solution was to a new volumetric flask, and the volume made up to $1 \mathrm{~mL}$ with methanol and the spectra were recorded on an Ultraspec 2000 UV/visible spectrophotometer (Pharmacia Biotech); MS data were recorded on a Bruker Esquire 3000 plus spectrometer. NMR spectra were recorded in $\mathrm{CDCl}_{3}$ (50.0 mg of 2-methoxy-1,4-naphthoquinone dissolved in $0.5 \mathrm{~mL}$ of solvent) on a Bruker Avance DMX $500 \mathrm{MHz}$ spectrometer operating at $500 \mathrm{MHz}\left({ }^{1} \mathrm{H}\right)$ or $125 \mathrm{MHz}\left({ }^{13} \mathrm{C}\right)$, respectively. Curcumin $(94 \%$ purity) was obtained from Sigma Chemical Co. (St. Louis, MO, USA). HPLC analyses were carried out on a Agilent 1100 series HPLC (Agilent Technologies, Palo Alto, CA, USA) equipped with a DAD 1100 diode array detector.

\section{Plant material}

Impatiens balsamina was grown in our lab and was authenticated by Professor Yao Zhensheng. The leaves of Impatiens balsamina were collected in August 2006, dried at room temperature for 2 weeks and then stored for further use.

\section{Preparation of extracts}

Dried leaves (200 g) were crushed, soaked with 55\% alcohol (1500 mL) for $24 \mathrm{~h}$ and then reflux extracted for $40 \mathrm{~min}(1500 \mathrm{~mL} \times 3)$. Extracts were filtered and vacuum evaporated; the residues were then suspended using distilled water and divided into five portions that were extracted systematically 
with the following solvents: petroleum ether (PEF), chloroform (CHF), ethyl acetate (EAF), $n$-butanol (BUF) and water (WAF). In addition, $200 \mathrm{~g}$ dried leaves were directly reflux extracted twice using chloroform (1500 mL/time). Next these extracts were filtered, combined, vacuum evaporated and the residue (CHE, $7.8 \mathrm{~g}$ ) was dried for further use.

\section{MTT test}

Human hepatocellular carcinoma cell line HepG2 purchased from the Cell Bank of The Chinese Academy of Science (Shanghai, P.R. China) was grown in RPMI-1640 medium (Gibco/Invitrogen Corp., Carlsbad, CA, USA) containing 10\% FCS, 100 units/mL penicillin (Sigma, St. Louis, MO, USA) and $50 \mu \mathrm{g} / \mathrm{mL}$ streptomycin (Sigma) and was incubated at $37^{\circ} \mathrm{C}$ in $5 \% \mathrm{CO}_{2}$. Cells in 96 -wells $\left(2 \times 10^{4}\right.$ cells/well) were treated with the different LIB fractions for $24 \mathrm{~h}$. All the extracts and fractions were dissolved in DMSO, and then diluted with RPMI-1640 medium to obtain the required concentration before use, and cells grown in medium containing an equivalent final volume of DMSO $(<0.1 \% \mathrm{v} / \mathrm{v})$ served as a vehicle control. At the end of treatment, $20 \mu \mathrm{L}$ of fresh serum-free medium containing MTT (5 mg/mL) reagent was added to each well. Cells were incubated for $4 \mathrm{~h}$ at $37^{\circ} \mathrm{C}$, and the medium was then carefully aspirated from the wells. Cells were lysed by addition of $200 \mu \mathrm{L}$ DMSO, and the optical density (OD) at $570 \mathrm{~nm}$ was determined using a Bio-Tek PowerWave 340 microplate scanning spectrophotometer. Data are reported as the average of at least three replicates.

\section{Differential display analysis of TLC spots}

Samples were dissolved in the appropriate organic solvent, applied to the silica gel G plates (10 cm $\times 20 \mathrm{~cm}$ activated at $105^{\circ} \mathrm{C}$ for $1 \mathrm{~h}$ ) and developed with several different suitable solvent systems (see details in the Results and Discussion section). The plates were dried in a fume hood, photographed under an ultraviolet lamp and then were further sprayed by reagents to better display the components from the different fractions.

\section{Additional fractionation of active fractions}

Active fractions or sub-fractions were further separated by silica gel chromatography, eluting with corresponding eluent, and monitored by TLC (see details in the Results and Discussion section).

\section{Active component identification}

A portion of sub-fraction CHE2 $(90.6 \mathrm{mg}$ ) was re-chromatographed on silica gel using a petroleum ether-acetone (8:2) system es eluent and the isolated product was recrystallized from $\mathrm{CHCl}_{3}$ to yield the active component as light yellow crystals, $88.1 \mathrm{mg}(97.2 \%)$. TLC confirmed that it was the dark spot in Figure 2-b, as marked by a black arrow. In addition, HPLC showed a single peak (date not shown). The compound was identified as 2-methoxy-1,4-naphthoquinone (MNQ), according to the following data: m.p. 183-183.5 $\mathrm{C}$; UV $\lambda_{\mathrm{MeOH}}(\log \varepsilon$ ) (Figure 5): 215 (3.95), 241 (4.21), 246 (4.21), 275 
(4.16), 329 (3.46); EIS-MS (Figure 6) m/z: $210.8[\mathrm{M}+\mathrm{Na}]{ }^{+} ;{ }^{13} \mathrm{C}-\mathrm{NMR}$ (Figure 7) $\delta$ : 180.3 (C-1), 160.6 (C-2), 110.1 (C-3), 185.0 (C-4), 126.9 (C-5), 134.5 (C-6), 133.5 (C-7), 126.4 (C-8), 131.3 (C-9), 132.2 (C-10), 56.6 (C-11); ${ }^{1} \mathrm{H}-\mathrm{NMR}$ (Figure 8) $\delta: 3.90$ (3H, s, H-2-OCH ${ }_{3}$ ), 6.16 (1H, s, H-3), 7.73 (2H, $\mathrm{m}, \mathrm{H}-5,8) 8.10$ (2H, m, H-6,7), which were all consistent with those described previously $[2,7,8]$.

Figure 5. UV spectrum of MNQ.

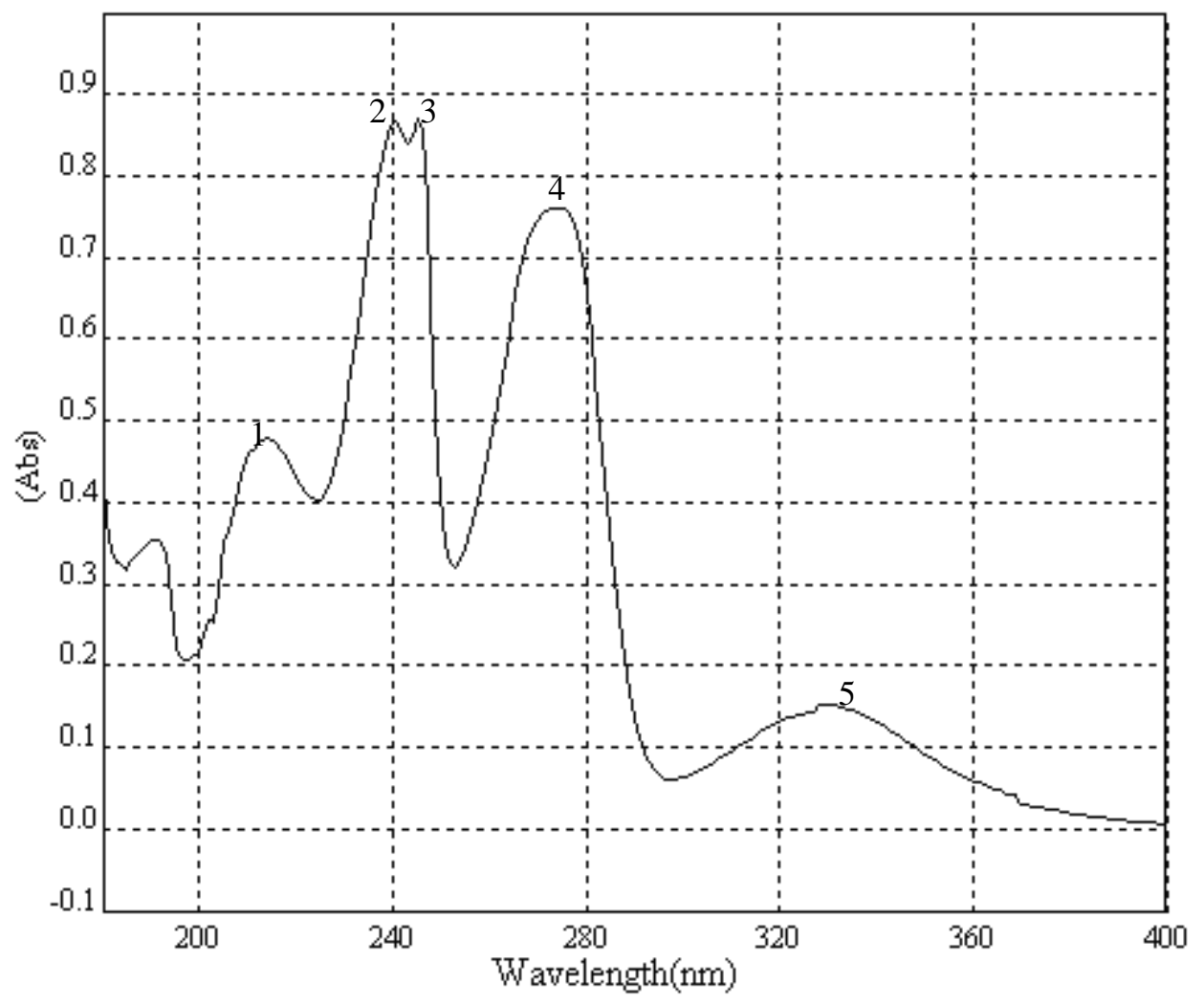

\begin{tabular}{cccl} 
Peak & $\boldsymbol{\lambda} \mathbf{n m}$ & Absorbance & \multicolumn{1}{c}{$\boldsymbol{\varepsilon}$} \\
\hline 1 & 215.0 & 0.478153 & 3.953725 \\
2 & 241.0 & 0.867875 & 4.212615 \\
3 & 246.0 & 0.869165 & 4.21326 \\
4 & 275.0 & 0.76158 & 4.155873 \\
5 & 329.0 & 0.152071 & 3.456204
\end{tabular}

Figure 6. Positive ion EIS-MS spectrum of MNQ.

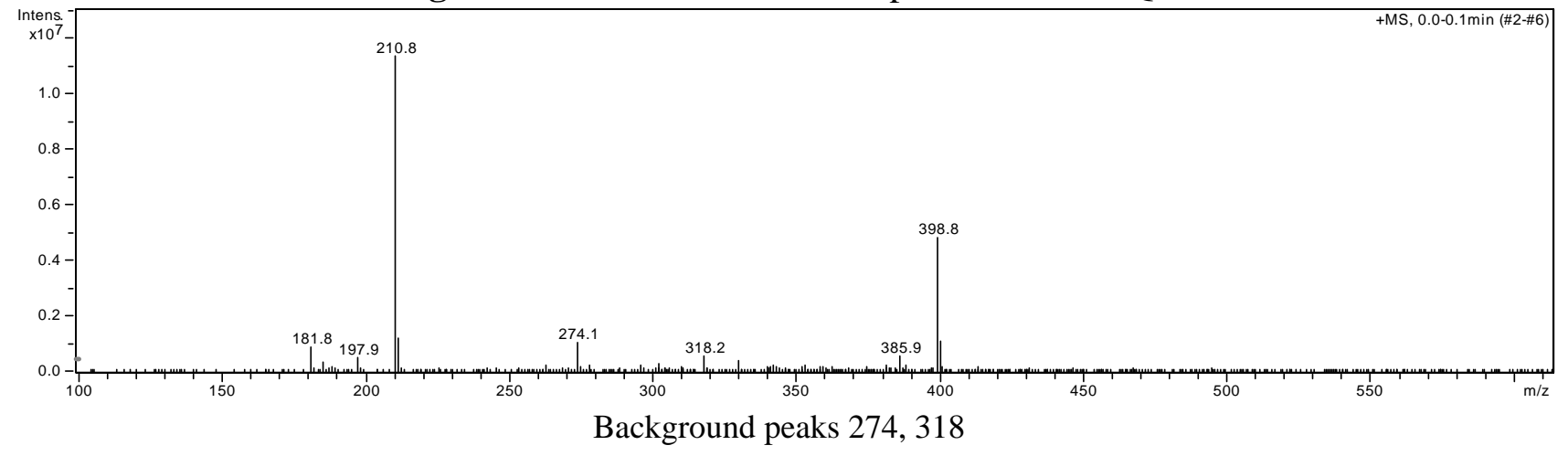


Figure $7 .{ }^{13} \mathrm{C}-\mathrm{NMR}$ spectrum of MNQ.

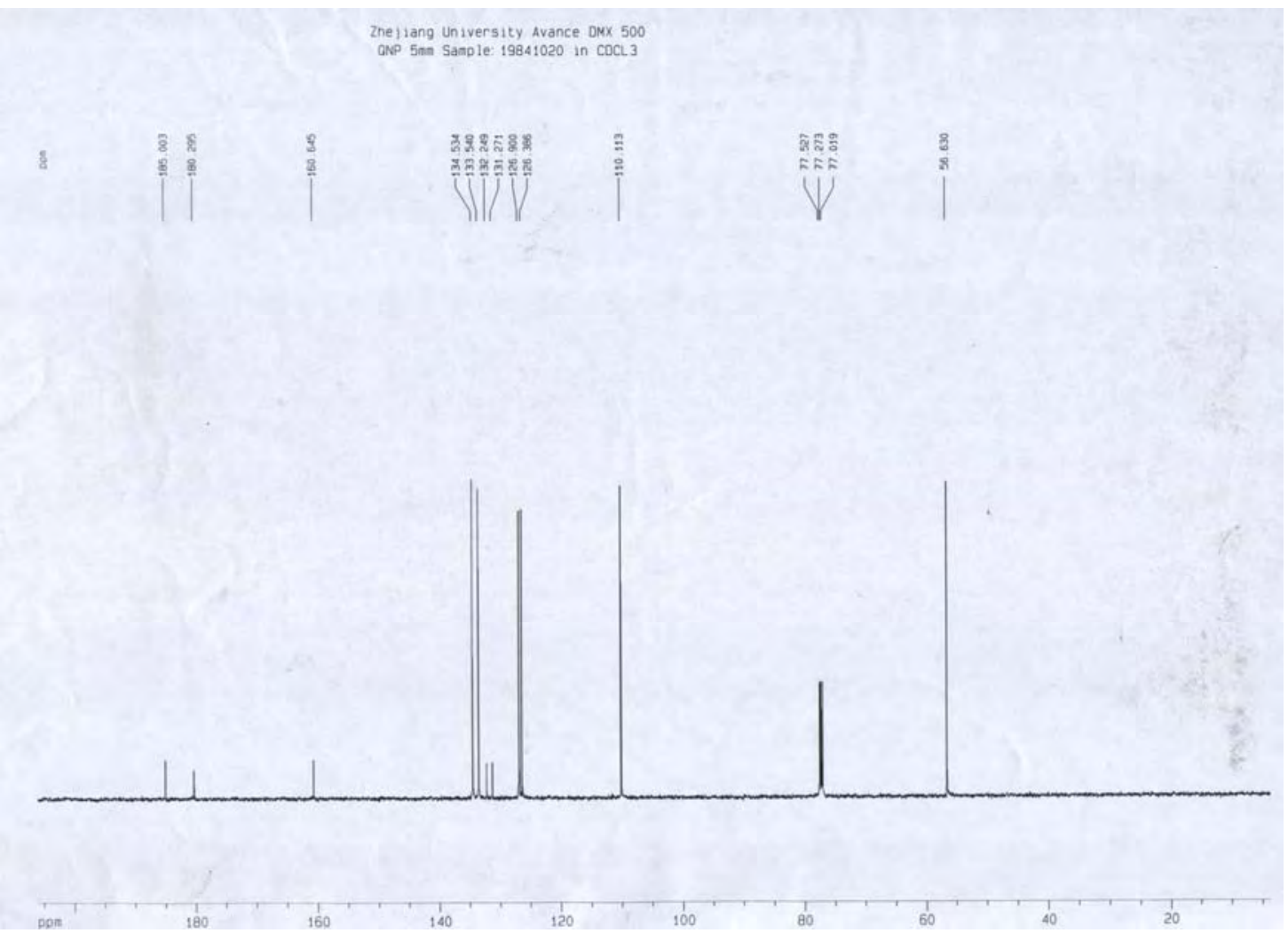

Figure 8. ${ }^{1} \mathrm{H}-\mathrm{NMR}$ spectrum of MNQ.

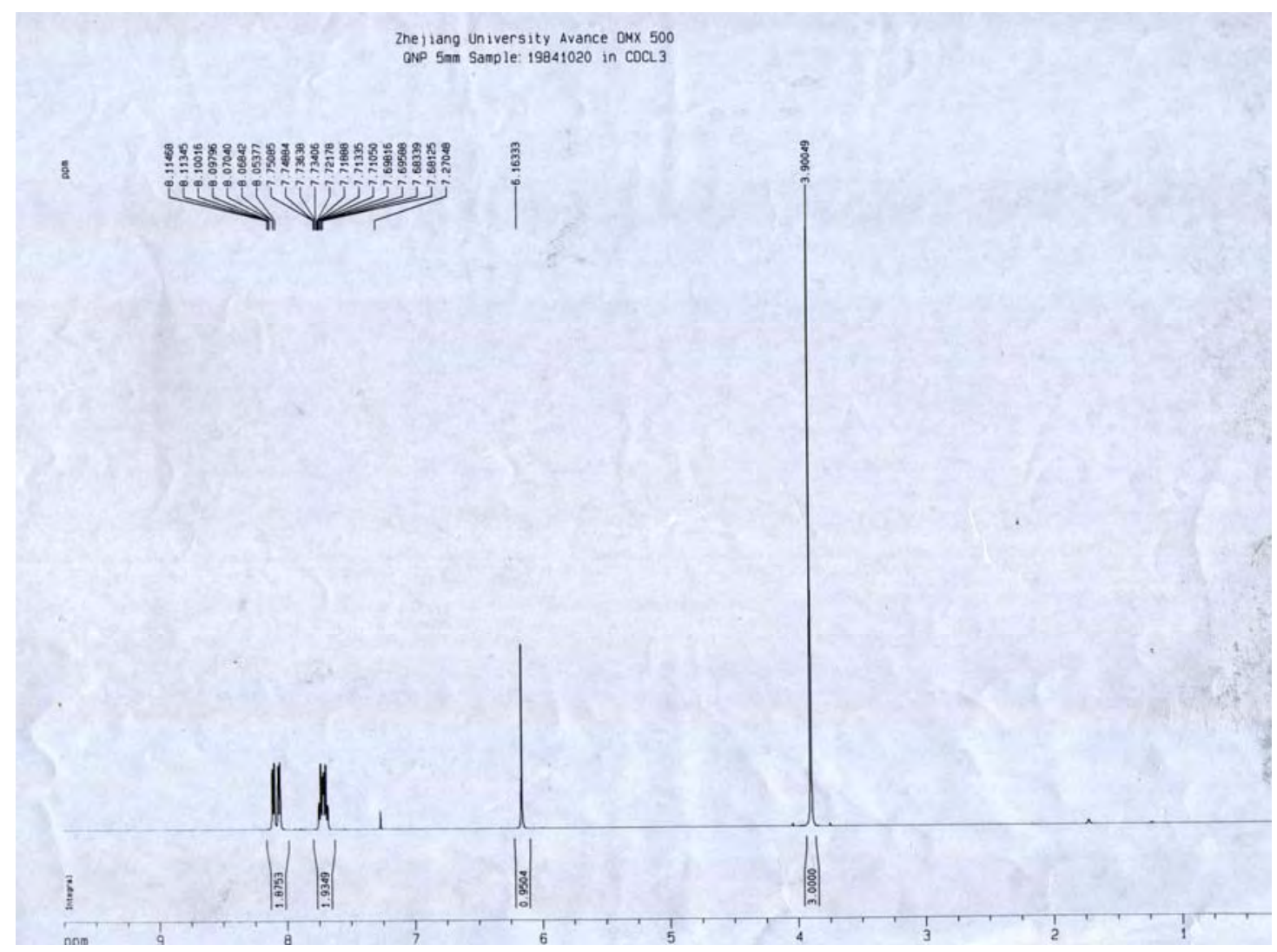




\section{References}

1. Jiang Su New Medicinal College. Dictionary of Chinese Crude Drugs. Shang Hai Scientific Technological Publishers: Shanghai, P.R. China, 2003.

2. Yang, X.; Summerhurst, D.K.; Koval, S.F.; Ficker, C.; Smith, M.L.; Bernards, M.A. Isolation of an antimicrobial compound from Impatiens balsamina L. using bioassay-guided fractionation. Phytother Res. 2001, 15, 676-680.

3. Yoshimi, U.; Hisae, O.; Munekazu, I.; Kyoko, I. Effects on Blood Pressure Decrease in Response to PAF of Impatiens textori MIQ. Biol. Pharm. Bull. 2003, 26, 1505-1507.

4. Ishiguro, K.; Fukumoto, H.; Osada, S.; Isoi, K.; Semma, M. A practical, reproducible measure of murine anaphylaxis: Blood pressure monitoring of anaphylaxis and effect of Impatiens balsamina L. Phytother. Res. 1994, 8, 301-304.

5. Hisae, O.; Kyoko, I. Cyclooxygenase-2 Inhibitory 1,4-Naphthoquinones from Impatiens balsamina L. Biol. Pharm. Bull. 2002, 25, 658-660.

6. Oku, H.; Ishiguro, K. Antipruritic and antidermatitic effect of extract and compounds of Impatiens balsamina L. in atopic dermatitis model NC mice. Phytother Res. 2001, 15, 506-510.

7. Ishiguro, K., Ohira, Y., Oku, H. Antipruritic dinaphthofuran-7,12-dione derivatives from the pericarp of Impatiens balsamina. J. Nat. Prod. 1998, 61, 1126-1129.

8. Chapelle, J.P. 2-Methoxy-1,4-naphthoquinone in Impatiens glandulifera and related species. Phytochemistry 1974, 13, 662.

9. Little, J.E.; Sproston, T.J.; Foote, M.W. Isolation and antifungal action of naturally occurring 2-methoxy-1,4-naphthoquinone. J. Biol. Chem. 1948, 174, 335-342.

10. Kang, S.C.; Moon, Y.H. Isolation and antimicrobial actibity of a naphthoquinones from Impatiens balsamina. Saengyak Hakoechi 1992, 23, 240-247.

11. Liu, H.B.; Cui, C.B.; Ren, H.; Gu, Q.Q. Research advances in the anticancer agents from natural naphthoquinones and their artificial derivatives. Nat. Prod. Res. Dev. 2005, 17, 104-107.

Sample Availability: Contact the authors.

(C) 2008 by MDPI (http://www.mdpi.org). Reproduction is permitted for noncommercial purposes. 